\title{
Measurement of Raman Optical Activity with High-Frequency Polarization Modulation
}

\author{
Carin R. Lightner, ${ }^{\dagger}$ Daniel Gisler, ${ }^{\dagger}$ Stefan A. Meyer,$^{\dagger}$ Hannah Niese,$^{\dagger}$ Robert C. Keitel,$^{\dagger}$ \\ and David J. Norris ${ }^{*},+$ \\ †Optical Materials Engineering Laboratory, Department of Mechanical and Process Engineering, \\ ETH Zurich, 8092 Zurich, Switzerland \\ Istituto Ricerche Solari Locarno (IRSOL), \\ Università della Svizzera italiana (USI), 6605 Locarno-Monti, Switzerland
}

\section{Contents}

Section

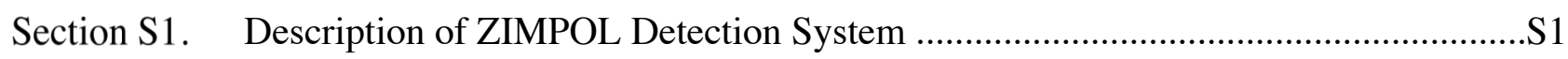

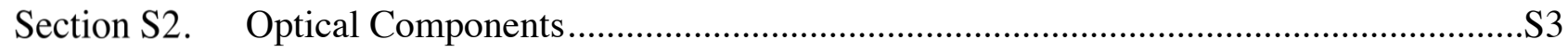

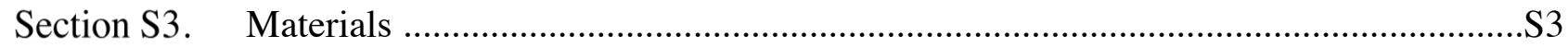

Section S4. Additional Control Measurements …......................................................................S4

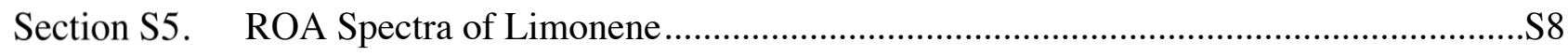

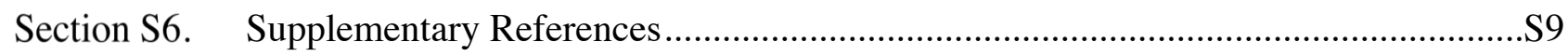

\section{Section S1. Description of ZIMPOL Detection System}

The Zurich Imaging Polarimeter (ZIMPOL) allows one to combine high-frequency polarization modulation with a CCD detector. ${ }^{\mathrm{S} 1}$ ZIMPOL operates by masking certain rows of the CCD detector and shifting the charges between rows with the polarization modulation frequency. This acts to essentially separate the different polarization states onto different rows of the detector. The basic principle is depicted in the cartoon plot in Figure S1. The modulation of light of different polarization states by the PEM is shown. In our instrument, the PEM modulation is set to a quarter-wave, and a linear polarizer behind the PEM is set at $+45^{\circ}$ to the optical axis of the PEM. The quarter-wave modulation means that circularly polarized light (CPL) incident onto the PEM is modulated at the operating frequency $(42 \mathrm{kHz})$. Light linearly polarized along the optical axis of the PEM is not 


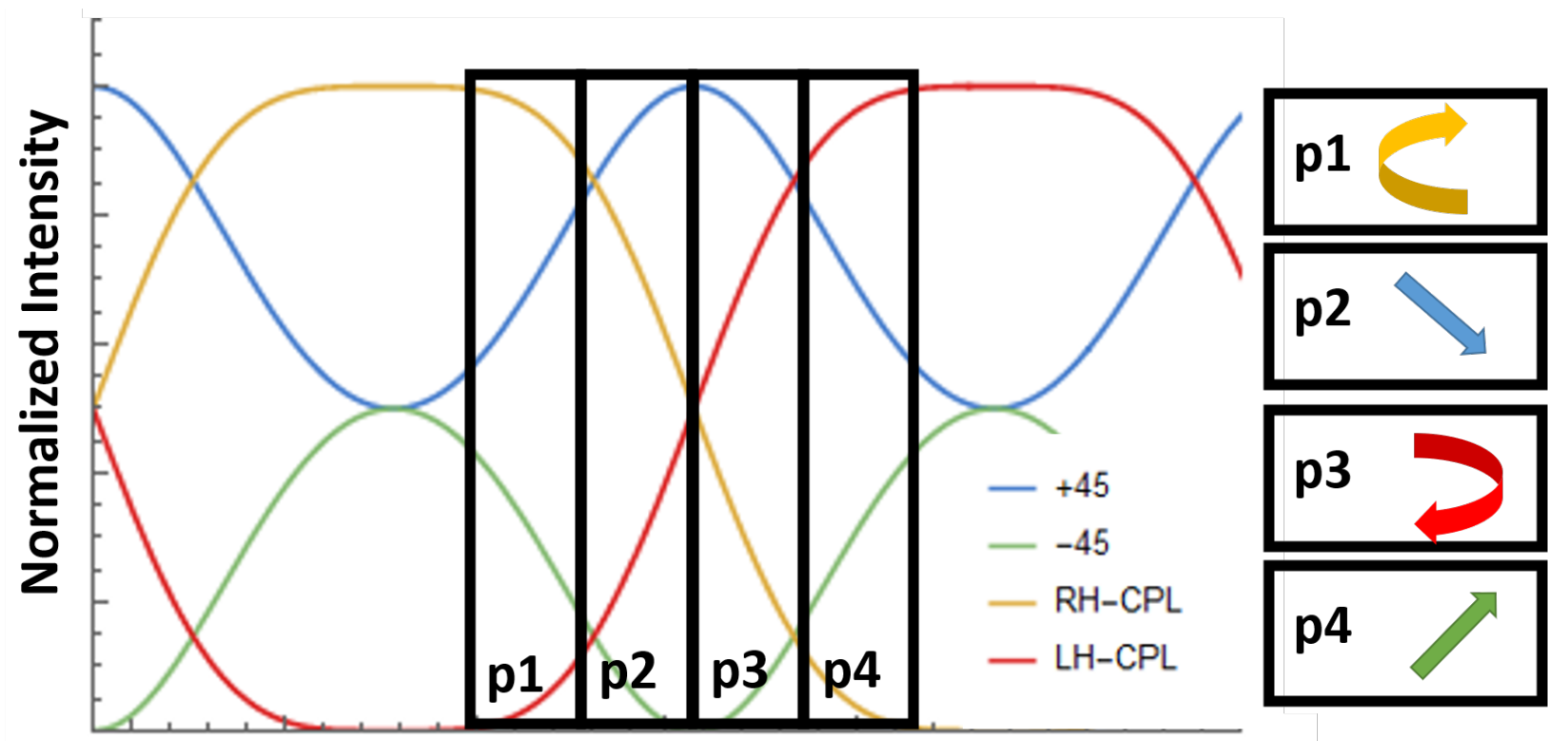

\section{Time}

Figure S1. The plot depicts the modulation of different polarization states by the PEM. As time progresses, ZIMPOL allows pixel rows p1, p2, p3, and p4 to detect the various polarization states (right).

modulated, but light linearly polarized at $45^{\circ}$ to the optical axis of the PEM is modulated at twice the operating frequency (shown as the blue and green curves in Figure S1).

By masking three out of four pixel rows, ZIMPOL effectively creates four images on the CCD which correspond to four time-steps in the PEM modulation. These time-steps (shown in Figure S1 as $\mathrm{p} 1, \mathrm{p} 2, \mathrm{p} 3$, and $\mathrm{p} 4$ ) can then be used to calculate the amount of left- versus right-handed CPL and plus and minus $45^{\circ}$ linearly polarized light in the beam incident on the PEM.

Because the working principle of ZIMPOL depends on shifting charge at high frequency, errors in this process can result in artifacts. Charge-shifting errors for each pixel lead to a fixed pattern of noise on the sensor, which is independent of time. To compensate for this noise, two images are taken and then subtracted from one another as part of the data processing. 
Section S2. Optical Components

\begin{tabular}{|l|l|l|}
\hline Component Name & Manufacturer & Part Number \\
\hline Glan-Taylor laser polarizer & Thor Labs & GL10 \\
\hline Zero-order 532 nm half-wave plate & Thor Labs & WPH10M-532 \\
\hline $100 \mathrm{~mm}$ focal distance focusing lens & Thor Labs & AC254-100-A-ML \\
\hline $30 \mathrm{~mm}$ focal distance collection lens & Thor Labs & AC254-030-A-ML \\
\hline $3 \mathrm{~mm}$ right-angle deflection mirror & Thor Labs & MRA03-P01 \\
\hline Photoelastic modulator & Hinds Instruments & II/FS42 \\
\hline Linear polarizer & Thor Labs & LPVISC100-MP2 \\
\hline Dielectric super notch plus Rayleigh filter & Iridian & DSNPF532-25 \\
\hline
\end{tabular}

Table S1. List of optical parts used in this work with manufacturer and part numbers.

\section{Section S3. Materials}

All chemicals were used neat (i.e. pure liquid) as received from the manufacturer. All chiral molecules were analytical standard with high enantiomeric purities. The manufacturer and purities are listed in Table S2 below.

\begin{tabular}{|l|l|c|}
\hline Chemical Name & Manufacturer & CAS Number \\
\hline Carbon tetrachloride $(99 \%)$ & Sigma Aldrich & $56-23-5$ \\
\hline Toluene $(\geq 99.5 \%)$ & Sigma Aldrich & $108-88-3$ \\
\hline$(+)-\alpha$-Pinene $[\geq 98.5 \%$ (sum of enantiomers, GC)] & Sigma Aldrich & $7785-70-8$ \\
\hline$(-)-\alpha$-Pinene $[\geq 99.0 \%$ (sum of enantiomers, GC)] & Sigma Aldrich & $7785-26-4$ \\
\hline$(+)-\beta$-Pinene $[\geq 98.5 \%$ (sum of enantiomers, GC)] & Sigma Aldrich & $19902-08-0$ \\
\hline$(-)-\beta$-Pinene $[\geq 98.5 \%$ (sum of enantiomers, GC)] & Sigma Aldrich & $18172-67-3$ \\
\hline$(+)$-Limonene $[\geq 99.0 \%$ (sum of enantiomers, GC)] & Sigma Aldrich & $5989-27-5$ \\
\hline$(-)$-Limonene $[\geq 99.0 \%$ (sum of enantiomers, GC)] & Sigma Aldrich & $5989-54-8$ \\
\hline
\end{tabular}

Table S2. List of chemicals used with manufacturer and CAS numbers. 


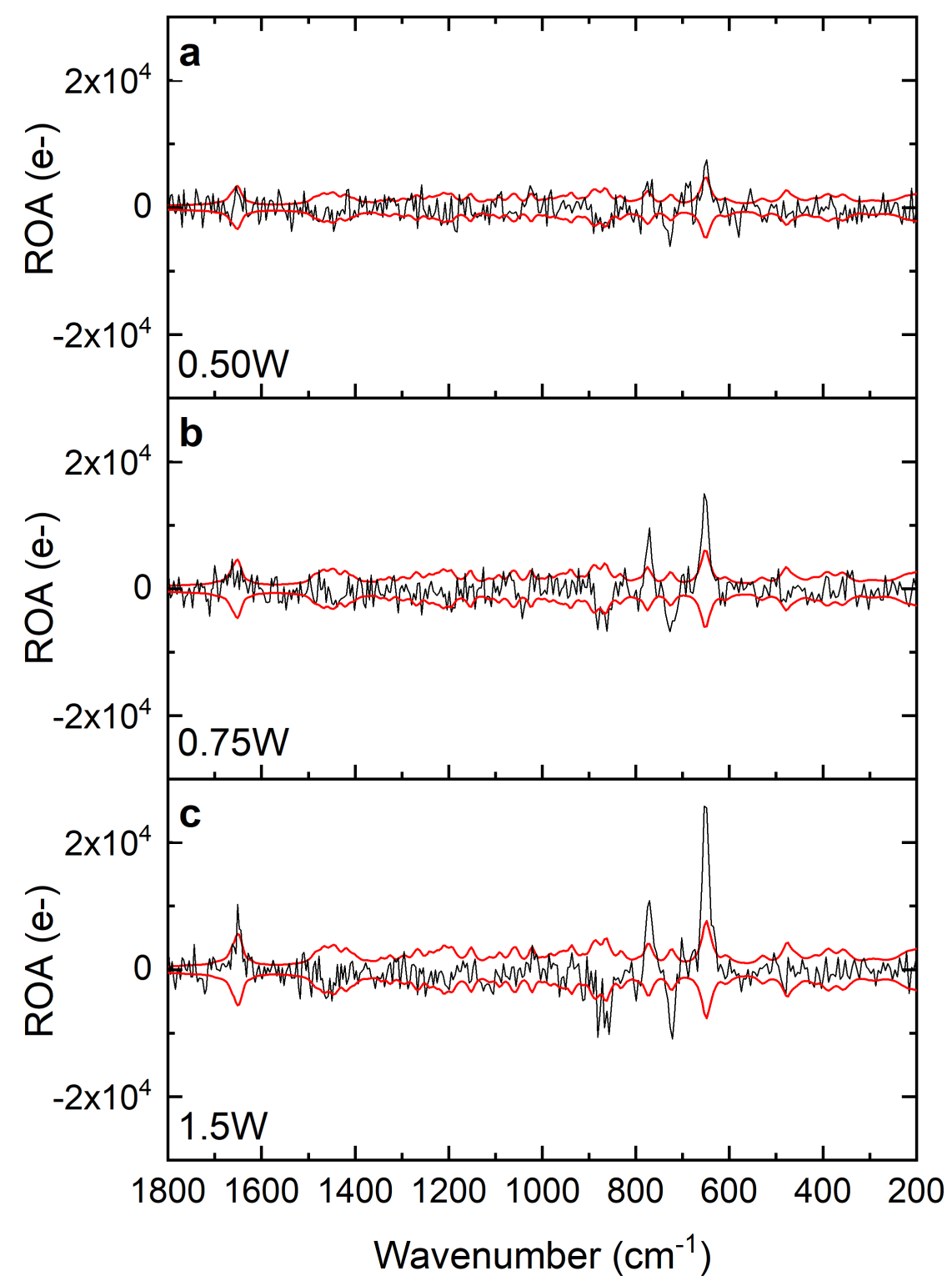

Figure S2. ROA spectra of (-)- $\beta$-Pinene (black curves) without phase correction, taken with different laser powers: (a) $0.50 \mathrm{~W}$, (b) $0.75 \mathrm{~W}$, and (c) $1.5 \mathrm{~W}$ with $3 \mathrm{~min}$ of accumulation time. The expected error from each measurement (square-root of the Raman signal) is shown in red. 


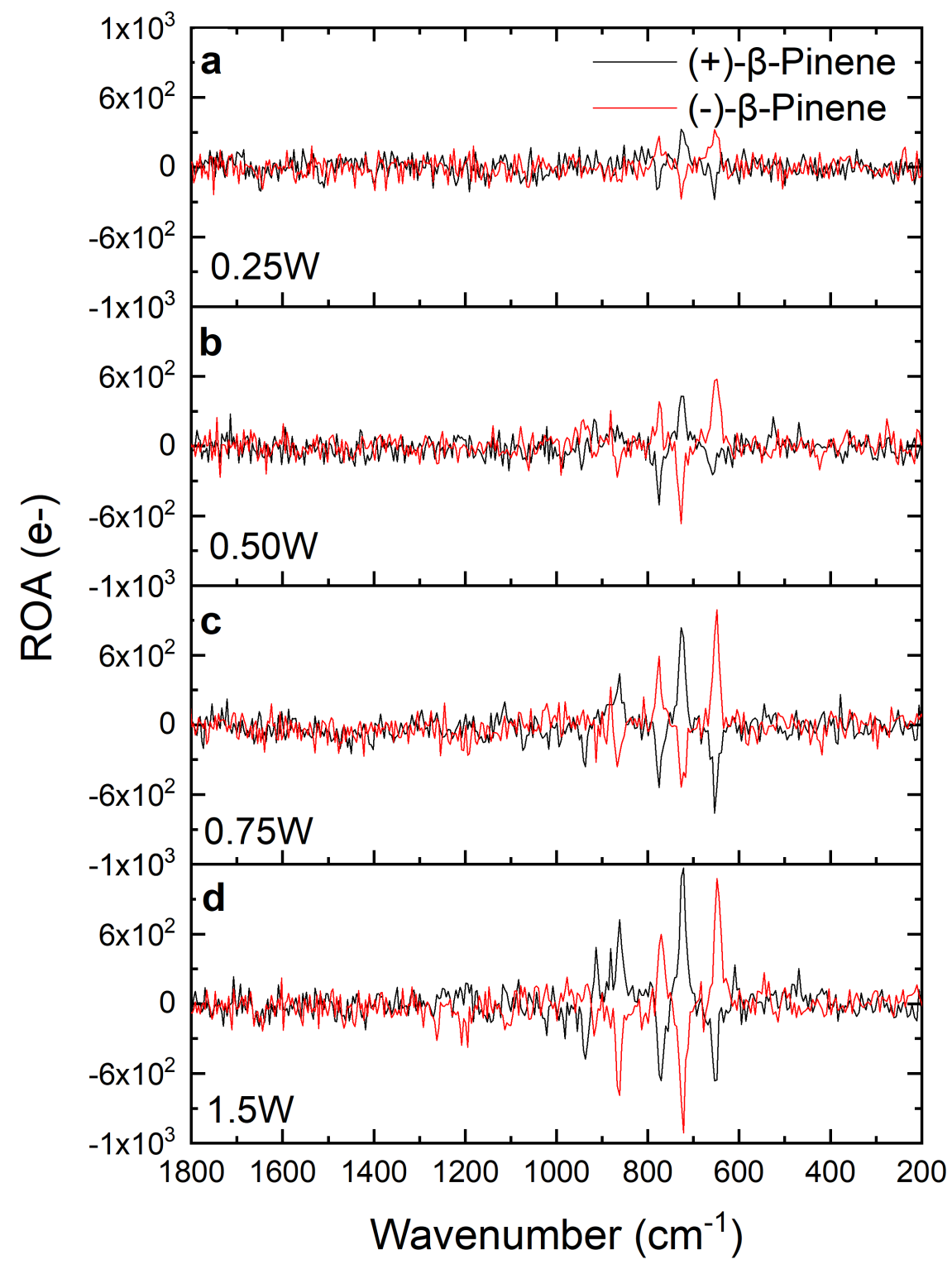

Figure S3. ROA spectra of (-)- $\beta$-pinene and $(+)-\beta$-pinene with two-phase correction, taken with different laser powers: (a) $0.25 \mathrm{~W}$, (b) $0.50 \mathrm{~W}$, (c) $0.75 \mathrm{~W}$, and (d) $1.5 \mathrm{~W}$ with 10 min of measurement time. 


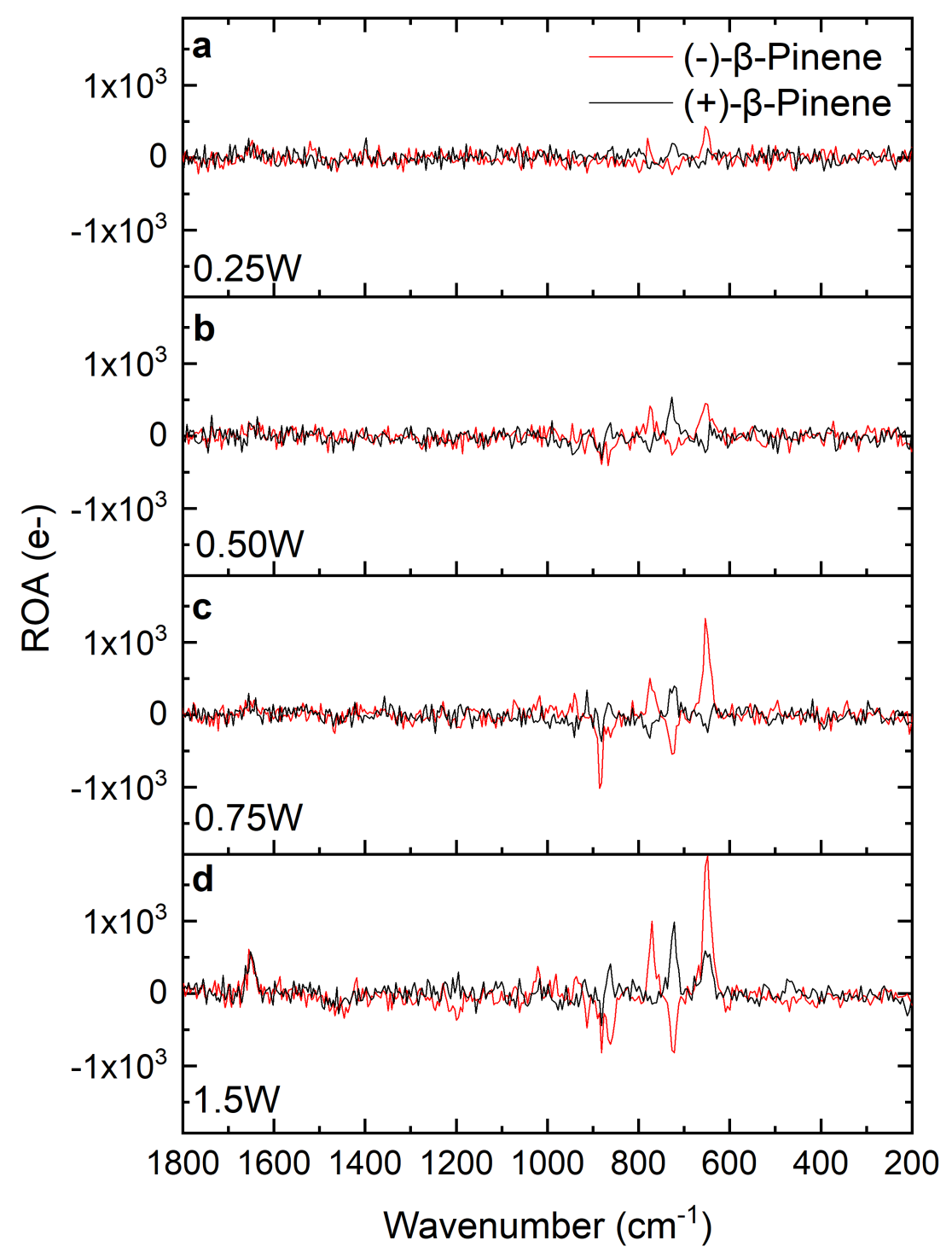

Figure S4. ROA spectra of (-)- $\beta$-pinene and (+)- $\beta$-pinene without phase correction, taken with different laser powers: (a) $0.25 \mathrm{~W}$, (b) $0.50 \mathrm{~W}$, (c) $0.75 \mathrm{~W}$, and (d) $1.5 \mathrm{~W}$ with 5 min of measurement time. 


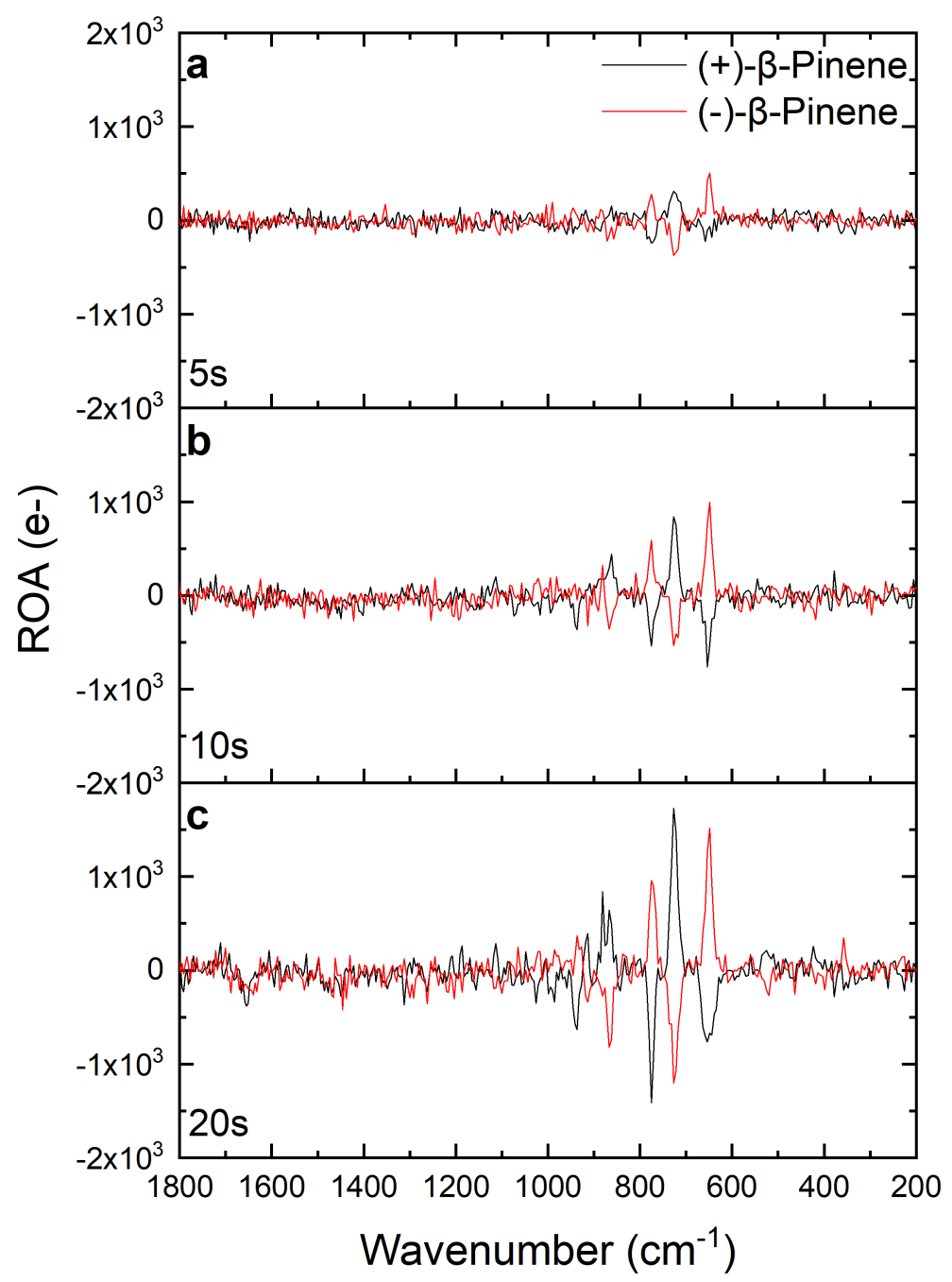

Figure S5. ROA spectra of (-)- $\beta$-pinene and $(+)-\beta$-pinene with two-phase correction, taken with different accumulation times: (a) $5 \mathrm{~s}$, (b) $10 \mathrm{~s}$, and (c) $20 \mathrm{~s}$ with $0.75 \mathrm{~W}$ of incident laser power and 20 accumulations. 


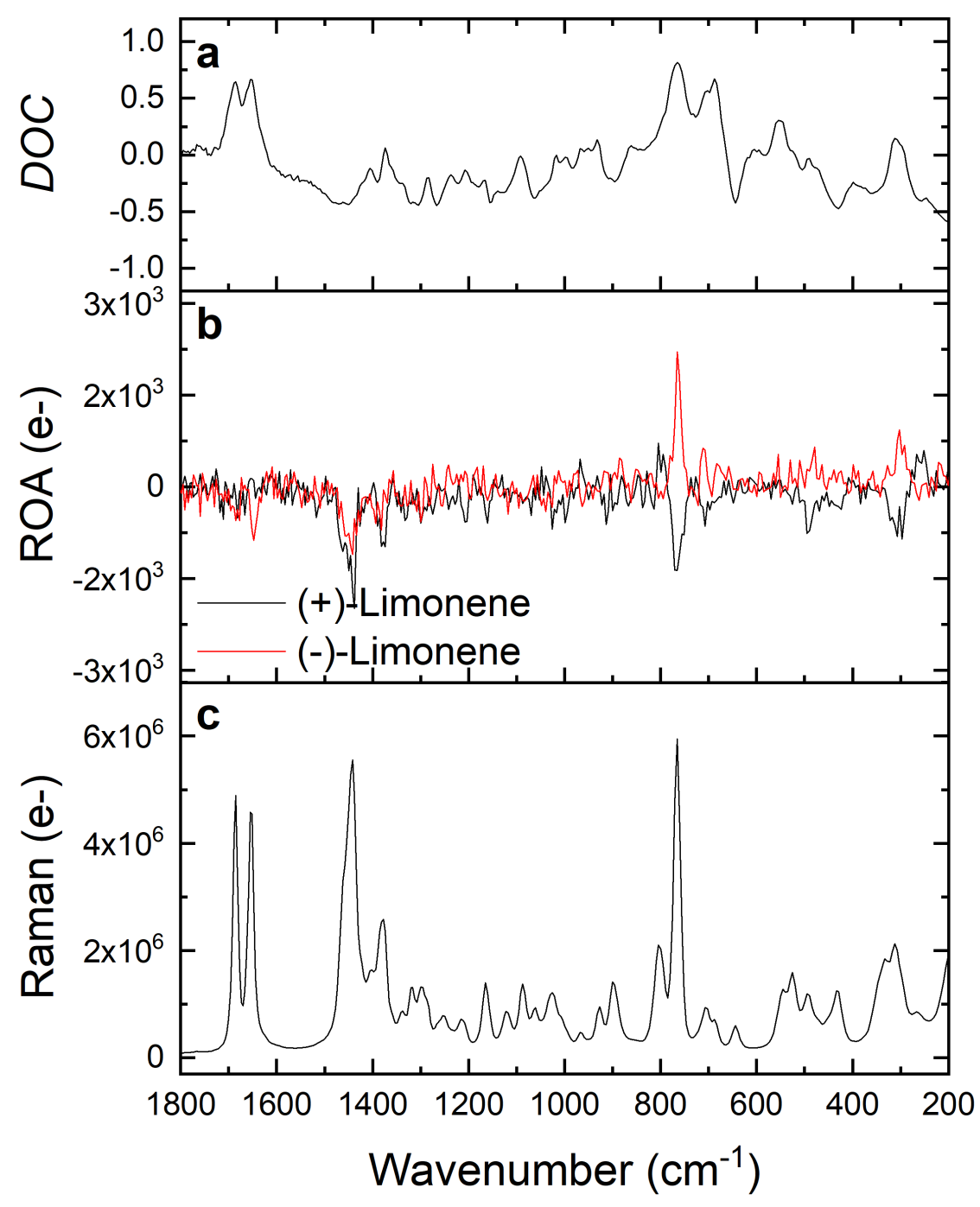

Figure S6. ROA spectra of limonene measured with $0.75 \mathrm{~W}$ of $532 \mathrm{~nm}$ laser light with $30 \mathrm{~min}$ of measurement time. (a) The DOC and (c) Raman spectra of (+)-limonene are shown for comparison with (b) the ROA spectra of $(+)$-limonene and (-)-limonene. 


\section{Section S6. Supplementary References}

S1. Povel, H.; Aebersold, H.; Stenflo, J. O., Charge-Coupled Device Image Sensor as a Demodulator in a 2-D Polarimeter with a Piezoelastic Modulator. Appl. Optics 1990, 29, 1186-1190. 